\title{
Learning Event Durations from Event Descriptions
}

\author{
Feng Pan, Rutu Mulkar, and Jerry R. Hobbs \\ Information Sciences Institute (ISI), University of Southern California \\ 4676 Admiralty Way, Marina del Rey, CA 90292, USA \\ \{pan, rutu, hobbs\}@isi.edu
}

\begin{abstract}
We have constructed a corpus of news articles in which events are annotated for estimated bounds on their duration. Here we describe a method for measuring inter-annotator agreement for these event duration distributions. We then show that machine learning techniques applied to this data yield coarse-grained event duration information, considerably outperforming a baseline and approaching human performance.
\end{abstract}

\section{Introduction}

Consider the sentence from a news article:

George W. Bush met with Vladimir Putin in Moscow.

How long was the meeting? Our first reaction to this question might be that we have no idea. But in fact we do have an idea. We know the meeting was longer than 10 seconds and less than a year. How much tighter can we get the bounds to be? Most people would say the meeting lasted between an hour and three days.

There is much temporal information in text that has hitherto been largely unexploited, encoded in the descriptions of events and relying on our knowledge of the range of usual durations of types of events. This paper describes one part of an exploration into how this information can be captured automatically. Specifically, we have developed annotation guidelines to minimize discrepant judgments and annotated 58 articles, comprising 2288 events; we have developed a method for measuring inter-annotator agreement when the judgments are intervals on a scale; and we have shown that machine learning techniques applied to the annotated data considerably out- perform a baseline and approach human performance.

This research is potentially very important in applications in which the time course of events is to be extracted from news. For example, whether two events overlap or are in sequence often depends very much on their durations. If a war started yesterday, we can be pretty sure it is still going on today. If a hurricane started last year, we can be sure it is over by now.

The corpus that we have annotated currently contains all the 48 non-Wall-Street-Journal (nonWSJ) news articles (a total of 2132 event instances), as well as 10 WSJ articles (156 event instances), from the TimeBank corpus annotated in TimeML (Pustejovky et al., 2003). The nonWSJ articles (mainly political and disaster news) include both print and broadcast news that are from a variety of news sources, such as $A B C$, $\mathrm{AP}$, and VOA.

In the corpus, every event to be annotated was already identified in TimeBank. Annotators were instructed to provide lower and upper bounds on the duration of the event, encompassing $80 \%$ of the possibilities, excluding anomalous cases, and taking the entire context of the article into account. For example, here is the graphical output of the annotations (3 annotators) for the "finished" event (underlined) in the sentence

After the victim, Linda Sanders, 35, had finished her cleaning and was waiting for her clothes to dry,...

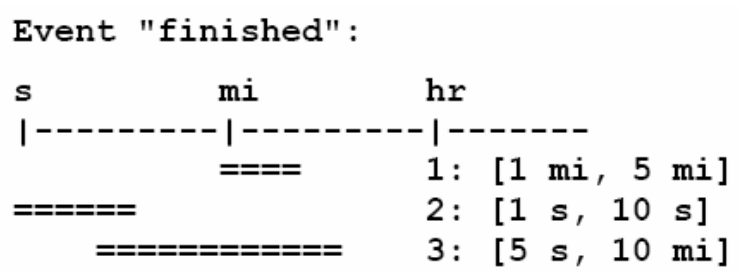


This graph shows that the first annotator believes that the event lasts for minutes whereas the second annotator believes it could only last for several seconds. The third annotates the event to range from a few seconds to a few minutes. A logarithmic scale is used for the output because of the intuition that the difference between 1 second and 20 seconds is significant, while the difference between 1 year 1 second and 1 year 20 seconds is negligible.

A preliminary exercise in annotation revealed about a dozen classes of systematic discrepancies among annotators' judgments. We thus developed guidelines to make annotators aware of these cases and to guide them in making the judgments. For example, many occurrences of verbs and other event descriptors refer to multiple events, especially but not exclusively if the subject or object of the verb is plural. In "Iraq has destroyed its long-range missiles", there is the time it takes to destroy one missile and the duration of the interval in which all the individual events are situated - the time it takes to destroy all its missiles. Initially, there were wide discrepancies because some annotators would annotate one value, others the other. Annotators are now instructed to make judgments on both values in this case. The use of the annotation guidelines resulted in about $10 \%$ improvement in inter-annotator agreement (Pan et al., 2006), measured as described in Section 2.

There is a residual of gross discrepancies in annotators' judgments that result from differences of opinion, for example, about how long a government policy is typically in effect. But the number of these discrepancies was surprisingly small.

The method and guidelines for annotation are described in much greater detail in (Pan et al., 2006). In the current paper, we focus on how inter-annotator agreement is measured, in Section 2, and in Sections 3-5 on the machine learning experiments. Because the annotated corpus is still fairly small, we cannot hope to learn to make fine-grained judgments of event durations that are currently annotated in the corpus, but as we demonstrate, it is possible to learn useful coarse-grained judgments.

Although there has been much work on temporal anchoring and event ordering in text (Hitzeman et al., 1995; Mani and Wilson, 2000; Filatova and Hovy, 2001; Boguraev and Ando, 2005), to our knowledge, there has been no serious published empirical effort to model and learn vague and implicit duration information in natu- ral language, such as the typical durations of events, and to perform reasoning over this information. (Cyc apparently has some fuzzy duration information, although it is not generally available; Rieger (1974) discusses the issue for less than a page; there has been work in fuzzy logic on representing and reasoning with imprecise durations (Godo and Vila, 1995; Fortemps, 1997), but these make no attempt to collect human judgments on such durations or learn to extract them automatically from texts.)

\section{Inter-Annotator Agreement}

Although the graphical output of the annotations enables us to visualize quickly the level of agreement among different annotators for each event, a quantitative measurement of the agreement is needed.

The kappa statistic (Krippendorff, 1980; Carletta, 1996) has become the de facto standard to assess inter-annotator agreement. It is computed as:

$$
\kappa=\frac{P(A)-P(E)}{1-P(E)}
$$

$P(A)$ is the observed agreement among the annotators, and $P(E)$ is the expected agreement, which is the probability that the annotators agree by chance.

In order to compute the kappa statistic for our task, we have to compute $P(A)$ and $P(E)$, but those computations are not straightforward.

$P(A)$ : What should count as agreement among annotators for our task?

$P(E)$ : What is the probability that the annotators agree by chance for our task?

\subsection{What Should Count as Agreement?}

Determining what should count as agreement is not only important for assessing inter-annotator agreement, but is also crucial for later evaluation of machine learning experiments. For example, for a given event with a known gold standard duration range from 1 hour to 4 hours, if a machine learning program outputs a duration of 3 hours to 5 hours, how should we evaluate this result?

In the literature on the kappa statistic, most authors address only category data; some can handle more general data, such as data in interval scales or ratio scales. However, none of the techniques directly apply to our data, which are ranges of durations from a lower bound to an upper bound. 


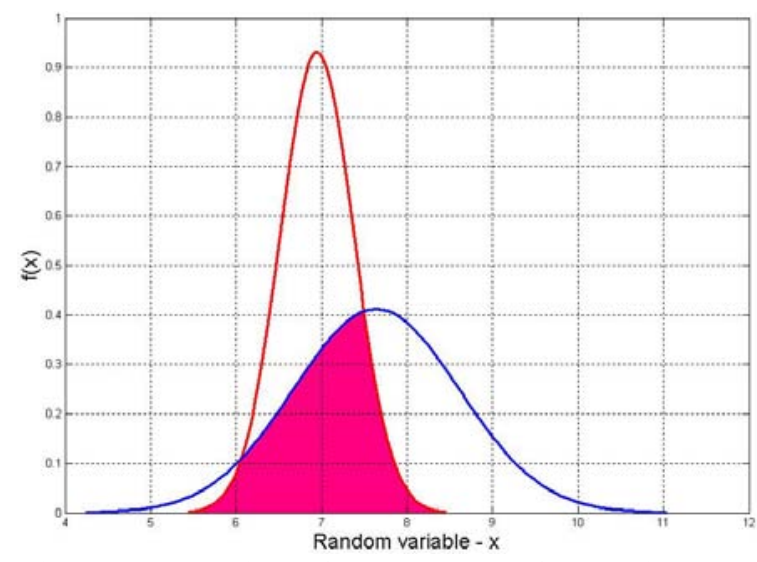

Figure 1: Overlap of Judgments of [10 minutes, 30 minutes] and [10 minutes, 2 hours].

In fact, what coders were instructed to annotate for a given event is not just a range, but a duration distribution for the event, where the area between the lower bound and the upper bound covers about $80 \%$ of the entire distribution area. Since it's natural to assume the most likely duration for such distribution is its mean (average) duration, and the distribution flattens out toward the upper and lower bounds, we use the normal or Gaussian distribution to model our duration distributions. If the area between lower and upper bounds covers $80 \%$ of the entire distribution area, the bounds are each 1.28 standard deviations from the mean.

Figure 1 shows the overlap in distributions for judgments of [10 minutes, 30 minutes] and [10 minutes, 2 hours], and the overlap or agreement is 0.508706 .

\subsection{Expected Agreement}

What is the probability that the annotators agree by chance for our task? The first quick response to this question may be 0 , if we consider all the possible durations from 1 second to 1000 years or even positive infinity.

However, not all the durations are equally possible. As in (Krippendorff, 1980), we assume there exists one global distribution for our task (i.e., the duration ranges for all the events), and "chance" annotations would be consistent with this distribution. Thus, the baseline will be an annotator who knows the global distribution and annotates in accordance with it, but does not read the specific article being annotated. Therefore, we must compute the global distribution of the durations, in particular, of their means and their widths. This will be of interest not only in determining expected agreement, but also in terms of

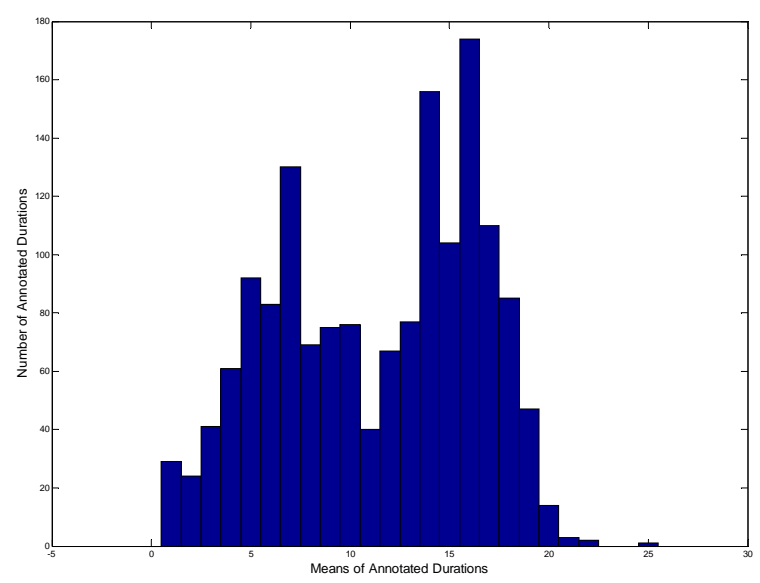

Figure 2: Distribution of Means of Annotated Durations.

what it says about the genre of news articles and about fuzzy judgments in general.

We first compute the distribution of the means of all the annotated durations. Its histogram is shown in Figure 2, where the horizontal axis represents the mean values in the natural logarithmic scale and the vertical axis represents the number of annotated durations with that mean.

There are two peaks in this distribution. One is from 5 to 7 in the natural logarithmic scale, which corresponds to about 1.5 minutes to 30 minutes. The other is from 14 to 17 in the natural logarithmic scale, which corresponds to about 8 days to 6 months. One could speculate that this bimodal distribution is because daily newspapers report short events that happened the day before and place them in the context of larger trends.

We also compute the distribution of the widths (i.e., $\mathrm{X}_{\text {upper }}-\mathrm{X}_{\text {lower }}$ ) of all the annotated durations, and its histogram is shown in Figure 3, where the horizontal axis represents the width in the natural logarithmic scale and the vertical axis represents the number of annotated durations with that width. Note that it peaks at about a half order of magnitude (Hobbs and Kreinovich, 2001).

Since the global distribution is determined by the above mean and width distributions, we can then compute the expected agreement, i.e., the probability that the annotators agree by chance, where the chance is actually based on this global distribution.

Two different methods were used to compute the expected agreement (baseline), both yielding nearly equal results. These are described in detail in (Pan et al., 2006). For both, P(E) is about 0.15. 


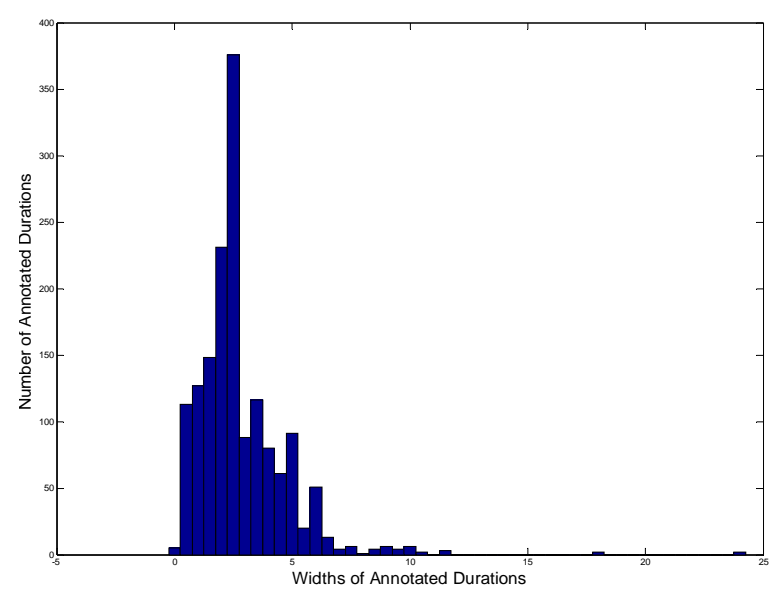

Figure 3: Distribution of Widths of Annotated Durations.

\section{Features}

In this section, we describe the lexical, syntactic, and semantic features that we considered in learning event durations.

\subsection{Local Context}

For a given event, the local context features include a window of $n$ tokens to its left and $n$ tokens to its right, as well as the event itself, for $n$ $=\{0,1,2,3\}$. The best $n$ determined via cross validation turned out to be 0 , i.e., the event itself with no local context. But we also present results for $n=2$ in Section 4.3 to evaluate the utility of local context.

A token can be a word or a punctuation mark. Punctuation marks are not removed, because they can be indicative features for learning event durations. For example, the quotation mark is a good indication of quoted reporting events, and the duration of such events most likely lasts for seconds or minutes, depending on the length of the quoted content. However, there are also cases where quotation marks are used for other purposes, such as emphasis of quoted words and titles of artistic works.

For each token in the local context, including the event itself, three features are included: the original form of the token, its lemma (or root form), and its part-of-speech (POS) tag. The lemma of the token is extracted from parse trees generated by the CONTEX parser (Hermjakob and Mooney, 1997) which includes rich context information in parse trees, and the Brill tagger (Brill, 1992) is used for POS tagging.

The context window doesn't cross the boundaries of sentences. When there are not enough tokens on either side of the event within the window, "NULL" is used for the feature values.

\begin{tabular}{|l|l|l|l|}
\hline Features & Original & Lemma & POS \\
\hline Event & signed & sign & VBD \\
\hline 1token-after & the & the & DT \\
\hline 2token-after & plan & plan & NN \\
\hline 1token-before & Friday & Friday & NNP \\
\hline 2token-before & on & on & IN \\
\hline
\end{tabular}

Table 1: Local context features for the "signed" event in sentence (1) with $n=2$.

The local context features extracted for the "signed" event in sentence (1) is shown in Table 1 (with a window size $n=2$ ). The feature vector is [signed, sign, VBD, the, the, DT, plan, plan, NN, Friday, Friday, NNP, on, on, IN].

(1) The two presidents on Friday signed the plan.

\subsection{Syntactic Relations}

The information in the event's syntactic environment is very important in deciding the durations of events. For example, there is a difference in the durations of the "watch" events in the phrases "watch a movie" and "watch a bird fly".

For a given event, both the head of its subject and the head of its object are extracted from the parse trees generated by the CONTEX parser. Similarly to the local context features, for both the subject head and the object head, their original form, lemma, and POS tags are extracted as features. When there is no subject or object for an event, "NULL" is used for the feature values.

For the "signed" event in sentence (1), the head of its subject is "presidents" and the head of its object is "plan". The extracted syntactic relation features are shown in Table 2, and the feature vector is [presidents, president, NNS, plan, plan, NN].

\subsection{WordNet Hypernyms}

Events with the same hypernyms may have similar durations. For example, events "ask" and "talk" both have a direct WordNet (Miller, 1990) hypernym of "communicate", and most of the time they do have very similar durations in the corpus.

However, closely related events don't always have the same direct hypernyms. For example, "see" has a direct hypernym of "perceive", whereas "observe" needs two steps up through the hypernym hierarchy before reaching "perceive". Such correlation between events may be lost if only the direct hypernyms of the words are extracted. 


\begin{tabular}{|l|l|l|l|}
\hline Features & Original & Lemma & POS \\
\hline Subject & presidents & president & NNS \\
\hline Object & plan & plan & NN \\
\hline
\end{tabular}

Table 2: Syntactic relation features for the "signed” event in sentence (1).

\begin{tabular}{|l|l|l|l|}
\hline Feature & 1-hyper & 2-hyper & 3-hyper \\
\hline Event & write & communicate & interact \\
\hline Subject & $\begin{array}{l}\text { corporate } \\
\text { executive }\end{array}$ & executive & $\begin{array}{l}\text { adminis- } \\
\text { trator }\end{array}$ \\
\hline Object & idea & content & cognition \\
\hline
\end{tabular}

Table 3: WordNet hypernym features for the event ("signed"), its subject ("presidents"), and its object ("plan”) in sentence (1).

It is useful to extract the hypernyms not only for the event itself, but also for the subject and object of the event. For example, events related to a group of people or an organization usually last longer than those involving individuals, and the hypernyms can help distinguish such concepts. For example, "society" has a "group" hypernym (2 steps up in the hierarchy), and "school" has an "organization" hypernym (3 steps up). The direct hypernyms of nouns are always not general enough for such purpose, but a hypernym at too high a level can be too general to be useful. For our learning experiments, we extract the first 3 levels of hypernyms from WordNet.

Hypernyms are only extracted for the events and their subjects and objects, not for the local context words. For each level of hypernyms in the hierarchy, it's possible to have more than one hypernym, for example, "see" has two direct hypernyms, "perceive" and "comprehend". For a given word, it may also have more than one sense in WordNet. In such cases, as in (Gildea and Jurafsky, 2002), we only take the first sense of the word and the first hypernym listed for each level of the hierarchy. A word disambiguation module might improve the learning performance. But since the features we need are the hypernyms, not the word sense itself, even if the first word sense is not the correct one, its hypernyms can still be good enough in many cases. For example, in one news article, the word "controller" refers to an air traffic controller, which corresponds to the second sense in WordNet, but its first sense (business controller) has the same hypernym of "person" (3 levels up) as the second sense (direct hypernym). Since we take the first 3 levels of hypernyms, the correct hypernym is still extracted.

\begin{tabular}{|c|c|c|}
\hline $\mathrm{P}(\mathrm{A})$ & $\mathrm{P}(\mathrm{E})$ & Kappa \\
\hline \multirow{2}{*}{$\mathbf{0 . 8 7 7}$} & 0.528 & 0.740 \\
\cline { 2 - 3 } & 0.500 & 0.755 \\
\hline
\end{tabular}

Table 4: Inter-Annotator Agreement for Binary Event Durations.

When there are less than 3 levels of hypernyms for a given word, its hypernym on the previous level is used. When there is no hypernym for a given word (e.g., "go"), the word itself will be used as its hypernyms. Since WordNet only provides hypernyms for nouns and verbs, "NULL" is used for the feature values for a word that is not a noun or a verb.

For the "signed" event in sentence (1), the extracted WordNet hypernym features for the event ("signed"), its subject ("presidents"), and its object ("plan") are shown in Table 3, and the feature vector is [write, communicate, interact, corporate_executive, executive, administrator, idea, content, cognition].

\section{Experiments}

The distribution of the means of the annotated durations in Figure 2 is bimodal, dividing the events into those that take less than a day and those that take more than a day. Thus, in our first machine learning experiment, we have tried to learn this coarse-grained event duration information as a binary classification task.

\subsection{Inter-Annotator Agreement, Baseline, and Upper Bound}

Before evaluating the performance of different learning algorithms, the inter-annotator agreement, the baseline and the upper bound for the learning task are assessed first.

Table 4 shows the inter-annotator agreement results among 3 annotators for binary event durations. The experiments were conducted on the same data sets as in (Pan et al., 2006). Two kappa values are reported with different ways of measuring expected agreement $(\mathrm{P}(\mathrm{E}))$, i.e., whether or not the annotators have prior knowledge of the global distribution of the task.

The human agreement before reading the guidelines (0.877) is a good estimate of the upper bound performance for this binary classification task. The baseline for the learning task is always taking the most probable class. Since $59.0 \%$ of the total data is "long" events, the baseline performance is $59.0 \%$. 


\begin{tabular}{|l|l|c|c|c|}
\hline Class & Algor. & Prec. & Recall & F-Score \\
\hline \multirow{3}{*}{ Short } & SVM & 0.707 & 0.606 & $\mathbf{0 . 6 5 3}$ \\
\cline { 2 - 5 } & NB & 0.567 & 0.768 & 0.652 \\
\cline { 2 - 5 } & C4.5 & 0.571 & 0.600 & 0.585 \\
\hline \multirow{3}{*}{ Long } & SVM & 0.793 & 0.857 & $\mathbf{0 . 8 2 3}$ \\
\cline { 2 - 5 } & NB & 0.834 & 0.665 & 0.740 \\
\cline { 2 - 5 } & C4.5 & 0.765 & 0.743 & 0.754 \\
\hline
\end{tabular}

Table 5: Test Performance of Three Algorithms.

\subsection{Data}

The original annotated data can be straightforwardly transformed for this binary classification task. For each event annotation, the most likely (mean) duration is calculated first by averaging (the logs of) its lower and upper bound durations. If its most likely (mean) duration is less than a day (about 11.4 in the natural logarithmic scale), it is assigned to the "short" event class, otherwise it is assigned to the "long" event class. (Note that these labels are strictly a convenience and not an analysis of the meanings of "short" and "long".)

We divide the total annotated non-WSJ data (2132 event instances) into two data sets: a training data set with 1705 event instances (about $80 \%$ of the total non-WSJ data) and a held-out test data set with 427 event instances (about 20\% of the total non-WSJ data). The WSJ data (156 event instances) is kept for further test purposes (see Section 4.4).

\subsection{Experimental Results (non-WSJ)}

Learning Algorithms. Three supervised learning algorithms were evaluated for our binary classification task, namely, Support Vector Machines (SVM) (Vapnik, 1995), Naïve Bayes (NB) (Duda and Hart, 1973), and Decision Trees C4.5 (Quinlan, 1993). The Weka (Witten and Frank, 2005) machine learning package was used for the implementation of these learning algorithms. Linear kernel is used for SVM in our experiments.

Each event instance has a total of 18 feature values, as described in Section 3, for the event only condition, and 30 feature values for the local context condition when $n=2$. For SVM and C4.5, all features are converted into binary features (6665 and 12502 features).

Results. 10-fold cross validation was used to train the learning models, which were then tested on the unseen held-out test set, and the performance (including the precision, recall, and F-score ${ }^{1}$

$1 \mathrm{~F}$-score is computed as the harmonic mean of the precision and recall: $\mathrm{F}=\left(2 * \mathrm{Prec}^{*} \mathrm{Rec}\right) /(\mathrm{Prec}+\mathrm{Rec})$.

\begin{tabular}{|l|l|}
\hline Algorithm & Precision \\
\hline Baseline & $59.0 \%$ \\
\hline C4.5 & $69.1 \%$ \\
\hline NB & $70.3 \%$ \\
\hline SVM & $\mathbf{7 6 . 6 \%}$ \\
\hline Human Agreement & $87.7 \%$ \\
\hline
\end{tabular}

Table 6: Overall Test Precision on non-WSJ Data.

for each class) of the three learning algorithms is shown in Table 5. The significant measure is overall precision, and this is shown for the three algorithms in Table 6, together with human agreement (the upper bound of the learning task) and the baseline.

We can see that among all three learning algorithms, SVM achieves the best F-score for each class and also the best overall precision (76.6\%). Compared with the baseline (59.0\%) and human agreement (87.7\%), this level of performance is very encouraging, especially as the learning is from such limited training data.

Feature Evaluation. The best performing learning algorithm, SVM, was then used to examine the utility of combinations of four different feature sets (i.e., event, local context, syntactic, and WordNet hypernym features). The detailed comparison is shown in Table 7.

We can see that most of the performance comes from event word or phrase itself. A significant improvement above that is due to the addition of information about the subject and object. Local context does not help and in fact may hurt, and hypernym information also does not seem to help ${ }^{2}$. It is of interest that the most important information is that from the predicate and arguments describing the event, as our linguistic intuitions would lead us to expect.

\subsection{Test on WSJ Data}

Section 4.3 shows the experimental results with the learned model trained and tested on the data with the same genre, i.e., non-WSJ articles.

In order to evaluate whether the learned model can perform well on data from different news genres, we tested it on the unseen WSJ data (156 event instances). The performance (including the precision, recall, and F-score for each class) is shown in Table 8 . The precision $(75.0 \%)$ is very close to the test performance on the non-WSJ

2 In the "Syn+Hyper" cases, the learning algorithm with and without local context gives identical results, probably because the other features dominate. 


\begin{tabular}{|c|c|c|c|c|c|c|c|c|c|}
\hline \multirow{2}{*}{ Class } & \multicolumn{3}{|c|}{ Event Only $(n=0)$} & \multicolumn{3}{|c|}{ Event Only + Syntactic } & \multicolumn{3}{|c|}{ Event + Syn + Hyper } \\
\hline & Prec. & Rec. & $\mathrm{F}$ & Prec. & Rec. & $\mathrm{F}$ & Prec. & Rec. & $\mathrm{F}$ \\
\hline Short & 0.742 & 0.465 & 0.571 & 0.758 & 0.587 & 0.662 & 0.707 & 0.606 & 0.653 \\
\hline Long & 0.748 & 0.908 & 0.821 & 0.792 & 0.893 & 0.839 & 0.793 & 0.857 & 0.823 \\
\hline Overall Prec. & \multicolumn{3}{|c|}{$74.7 \%$} & \multicolumn{3}{|c|}{$78.2 \%$} & \multicolumn{3}{|c|}{$76.6 \%$} \\
\hline & \multicolumn{3}{|c|}{ Local Context $(n=2)$} & \multicolumn{3}{|c|}{ Context + Syntactic } & \multicolumn{3}{|c|}{ Context + Syn + Hyper } \\
\hline Short & 0.672 & 0.568 & 0.615 & 0.710 & 0.600 & 0.650 & 0.707 & 0.606 & 0.653 \\
\hline Long & 0.774 & 0.842 & 0.806 & 0.791 & 0.860 & 0.824 & 0.793 & 0.857 & 0.823 \\
\hline Overall Prec. & \multicolumn{3}{|c|}{$74.2 \%$} & \multicolumn{3}{|c|}{$76.6 \%$} & \multicolumn{3}{|c|}{$76.6 \%$} \\
\hline
\end{tabular}

Table 7: Feature Evaluation with Different Feature Sets using SVM.

\begin{tabular}{|l|c|c|c|}
\hline Class & Prec. & Rec. & F \\
\hline Short & 0.692 & 0.610 & 0.649 \\
\hline Long & 0.779 & 0.835 & 0.806 \\
\hline Overall Prec. & \multicolumn{3}{|c|}{$\mathbf{7 5 . 0 \%}$} \\
\hline
\end{tabular}

Table 8: Test Performance on WSJ data.

\begin{tabular}{|c|c|c|}
\hline $\mathrm{P}(\mathrm{A})$ & $\mathrm{P}(\mathrm{E})$ & Kappa \\
\hline \multirow{2}{*}{$\mathbf{0 . 7 9 8}$} & 0.151 & 0.762 \\
\cline { 2 - 3 } & 0.143 & 0.764 \\
\hline
\end{tabular}

Table 9: Inter-Annotator Agreement for Most Likely Temporal Unit.

data, and indicates the significant generalization capacity of the learned model.

\section{Learning the Most Likely Temporal Unit}

These encouraging results have prompted us to try to learn more fine-grained event duration information, viz., the most likely temporal units of event durations (cf. (Rieger 1974)'s ORDERHOURS, ORDERDAYS).

For each original event annotation, we can obtain the most likely (mean) duration by averaging its lower and upper bound durations, and assigning it to one of seven classes (i.e., second, minute, hour, day, week, month, and year) based on the temporal unit of its most likely duration.

However, human agreement on this more finegrained task is low (44.4\%). Based on this observation, instead of evaluating the exact agreement between annotators, an "approximate agreement" is computed for the most likely temporal unit of events. In "approximate agreement", temporal units are considered to match if they are the same temporal unit or an adjacent one. For example, "second" and "minute" match, but "minute" and "day" do not.

Some preliminary experiments have been conducted for learning this multi-classification task. The same data sets as in the binary classification task were used. The only difference is that the class for each instance is now labeled with one

\begin{tabular}{|l|l|}
\hline Algorithm & Precision \\
\hline Baseline & $51.5 \%$ \\
\hline C4.5 & $56.4 \%$ \\
\hline NB & $65.8 \%$ \\
\hline SVM & $\mathbf{6 7 . 9 \%}$ \\
\hline Human Agreement & $79.8 \%$ \\
\hline
\end{tabular}

Table 10: Overall Test Precisions.

of the seven temporal unit classes.

The baseline for this multi-classification task is always taking the temporal unit which with its two neighbors spans the greatest amount of data. Since the "week", "month", and "year" classes together take up largest portion (51.5\%) of the data, the baseline is always taking the "month" class, where both "week" and "year" are also considered a match. Table 9 shows the interannotator agreement results for most likely temporal unit when using "approximate agreement". Human agreement (the upper bound) for this learning task increases from $44.4 \%$ to $79.8 \%$.

10 -fold cross validation was also used to train the learning models, which were then tested on the unseen held-out test set. The performance of the three algorithms is shown in Table 10. The best performing learning algorithm is again SVM with $67.9 \%$ test precision. Compared with the baseline (51.5\%) and human agreement (79.8\%), this again is a very promising result, especially for a multi-classification task with such limited training data. It is reasonable to expect that when more annotated data becomes available, the learning algorithm will achieve higher performance when learning this and more fine-grained event duration information.

Although the coarse-grained duration information may look too coarse to be useful, computers have no idea at all whether a meeting event takes seconds or centuries, so even coarse-grained estimates would give it a useful rough sense of how long each event may take. More fine-grained duration information is definitely more desirable for temporal reasoning tasks. But coarse-grained 
durations to a level of temporal units can already be very useful.

\section{Conclusion}

In the research described in this paper, we have addressed a problem -- extracting information about event durations encoded in event descriptions -- that has heretofore received very little attention in the field. It is information that can have a substantial impact on applications where the temporal placement of events is important. Moreover, it is representative of a set of problems - making use of the vague information in text - that has largely eluded empirical approaches in the past. In (Pan et al., 2006), we explicate the linguistic categories of the phenomena that give rise to grossly discrepant judgments among annotators, and give guidelines on resolving these discrepancies. In the present paper, we describe a method for measuring inter-annotator agreement when the judgments are intervals on a scale; this should extend from time to other scalar judgments. Inter-annotator agreement is too low on fine-grained judgments. However, for the coarse-grained judgments of more than or less than a day, and of approximate agreement on temporal unit, human agreement is acceptably high. For these cases, we have shown that machine-learning techniques achieve impressive results.

\section{Acknowledgments}

This work was supported by the Advanced Research and Development Activity (ARDA), now the Disruptive Technology Office (DTO), under DOD/DOI/ARDA Contract No. NBCHC040027. The authors have profited from discussions with Hoa Trang Dang, Donghui Feng, Kevin Knight, Daniel Marcu, James Pustejovsky, Deepak Ravichandran, and Nathan Sobo.

\section{References}

B. Boguraev and R. K. Ando. 2005. TimeMLCompliant Text Analysis for Temporal Reasoning. In Proceedings of International Joint Conference on Artificial Intelligence (IJCAI).

E. Brill. 1992. A simple rule-based part of speech tagger. In Proceedings of the Third Conference on Applied Natural Language Processing.

J. Carletta. 1996. Assessing agreement on classification tasks: the kappa statistic. Computational Lingustics, 22(2):249-254.

R. O. Duda and P. E. Hart. 1973. Pattern Classification and Scene Analysis. Wiley, New York.
E. Filatova and E. Hovy. 2001. Assigning TimeStamps to Event-Clauses. Proceedings of ACL Workshop on Temporal and Spatial Reasoning.

P. Fortemps. 1997. Jobshop Scheduling with Imprecise Durations: A Fuzzy Approach. IEEE Transactions on Fuzzy Systems Vol. 5 No. 4.

D. Gildea and D. Jurafsky. 2002. Automatic Labeling of Semantic Roles. Computational Linguistics, 28(3):245-288.

L. Godo and L. Vila. 1995. Possibilistic Temporal Reasoning based on Fuzzy Temporal Constraints. In Proceedings of International Joint Conference on Artificial Intelligence (IJCAI).

U. Hermjakob and R. J. Mooney. 1997. Learning Parse and Translation Decisions from Examples with Rich Context. In Proceedings of the 35th Annual Meeting of the Association for Computational Linguistics (ACL).

J. Hitzeman, M. Moens, and C. Grover. 1995. Algorithms for Analyzing the Temporal Structure of Discourse. In Proceedings of EACL. Dublin, Ireland.

J. R. Hobbs and V. Kreinovich. 2001. Optimal Choice of Granularity in Commonsense Estimation: Why Half Orders of Magnitude, In Proceedings of Joint 9th IFSA World Congress and 20th NAFIPS International Conference, Vacouver, British Columbia.

K. Krippendorf. 1980. Content Analysis: An introduction to its methodology. Sage Publications.

I. Mani and G. Wilson. 2000. Robust Temporal Processing of News. In Proceedings of the 38th Annual Meeting of the Association for Computational Linguistics (ACL).

G. A. Miller. 1990. WordNet: an On-line Lexical Database. International Journal of Lexicography 3(4).

F. Pan, R. Mulkar, and J. R. Hobbs. 2006. An Annotated Corpus of Typical Durations of Events. In Proceedings of the Fifth International Conference on Language Resources and Evaluation (LREC), Genoa, Italy.

J. Pustejovsky, P. Hanks, R. Saurí, A. See, R. Gaizauskas, A. Setzer, D. Radev, B. Sundheim, D. Day, L. Ferro and M. Lazo. 2003. The timebank corpus. In Corpus Linguistics, Lancaster, U.K.

J. R. Quinlan. 1993. C4.5: Programs for Machine Learning. Morgan Kaufmann, San Francisco.

C. J. Rieger. 1974. Conceptual memory: A theory and computer program for processing and meaning content of natural language utterances. Stanford AIM-233.

V. Vapnik. 1995. The Nature of Statistical Learning Theory. Springer-Verlag, New York.

I. H. Witten and E. Frank. 2005. Data Mining: Practical machine learning tools and techniques, 2nd Edition, Morgan Kaufmann, San Francisco. 